\title{
Editorial
}

\section{Should We Always Defer Treatment of Kidney Disease When There Is Extensive Interstitial Fibrosis on Biopsy?}

\author{
Musab S. Hommos Andrew D. Rule \\ Division of Nephrology and Hypertension, Mayo Clinic, Rochester, Minn., USA
}

\section{Key Words}

Kidney - Renal biopsy - Interstitial fibrosis - Tubular atrophy . Prognosis · Outcomes

\begin{abstract}
Severity of interstitial fibrosis and tubular atrophy (IFTA) seen on kidney biopsy has been used by nephrologist as prognostic marker of kidney disease. While IFTA is a very strong predictor of kidney failure, some patients with severe fibrosis will still not progress to kidney failure within 5 years. Treatment of underlying kidney disease in select patients with severe IFTA should be considered when risks of treatment are reasonable despite the low potential for benefit.
\end{abstract}

๑) 2016 S. Karger AG, Basel

Interstitial fibrosis and tubular atrophy (IFTA) is a common final pathway for chronic kidney disease (CKD) [1]. Severity of IFTA seen on kidney biopsy has long been used to judge the chronicity of kidney disease in order to predict renal outcomes and to balance risks and benefits of using immunomodulatory medications. IFTA severity is incorporated into a few scoring systems including the Oxford Classification for IgA nephropathy and Banff classification of renal allograft biopsies [2, 3]. However, impor-

\section{KARGER}

E-Mail karger@karger.com

www.karger.com/ajn tant questions remain with the application of IFTA severity to clinical care. While interstitial fibrosis has important prognostic values, is it sufficient for determining prognosis and making treatment decisions? In particular, is there a severity of IFTA at which treatment of the underlying kidney disease is no longer worth pursuing? To what extent does kidney function and other clinical characteristics contribute to prognosis beyond interstitial fibrosis?

In this issue of American Journal of Nephrology, Menn-Josephy et al. [4] attempt to answer these questions on 434 native kidney biopsies by relating the amount of IFTA to the risk of kidney failure requiring dialysis. They found IFTA was a good, albeit imperfect, predictor of end-stage renal disease $(E S R D ; C$-statistic $=0.832)$. Adding clinical characteristics improved the prediction of ESRD (C-statistic $=0.879)$; lower body mass index, higher serum creatinine, higher proteinuria, hypertension and insulin dependent diabetes mellitus were the other independent predictors of ESRD. However, models that predict the future are imperfect as demonstrated by this study. The authors extensively detail how patients with severe fibrosis $(>50 \%)$ could still have normal renal function and $21 \%$ with severe fibrosis did not progress to ESRD within 5 years. Based on these findings, they suggest that some patients with severe fibrosis may still benefit from treatment. 
This study illustrates important points about the application of biopsy fibrosis to the clinical care of individual patients. Even highly predictive findings such as IFTA are never perfect at predicting outcomes in individual patients. While a finding of severe fibrosis suggests that there is likely to be little benefit with treatment, treatment may still be worth pursuing if the risks of the treatment are small. For example, a young patient may present with untreated lupus nephritis and severe fibrosis on biopsy. Treatment with mycophenolate mofetil is generally well tolerated and worth consideration even if the patient is still likely to progress to ESRD. On the other hand, a patient with a treatment-resistant glomerulonephritis complicated by infections may not be a good candidate for further immunosuppressive therapy once severe fibrosis is evident on biopsy. Clinical trials should be used to help inform these decisions, but as pointed out by Menn-Josephy et al. [4], patients with severe fibrosis are often excluded from clinical trials.

There are several reasons why IFTA is not a perfect predictor of ESRD. First, CKD is often characterized by a patchy fibrosis. A random biopsy can over- or underestimate the true severity of IFTA for the entire kidney. Second, IFTA simply reflects destroyed non-functioning parenchyma. It is less informative about the primary underlying disease process. Treating the underlying kidney disease may still delay the rate of progression. Third, while fibrosis is a potent predictor of ESRD, kidney function markers such as lower serum creatinine and less proteinuria and the absence of the major risk factors for ESRD (hypertension and diabetes mellitus) are independently associated with a better renal prognosis. Thus, the amount of fibrosis on biopsy should not completely replace consideration of kidney function and risk factors when considering patient prognosis.

Subgroup analysis in this study provided additional findings. While fibrosis performed well in predicting who would progress to ESRD, it was not a statistically significant predictor of progression to ESRD in patients over 70 years of age or in patients with diabetic nephropathy. However, the statistical approach that was used to support this finding may not have been optimal. First, there may not be enough statistical power with subgroup analysis to detect associations seen in the overall sample. Notably, only 31 of the patients were over 70 years of age and only 42 had diabetic nephropathy. Second, the more appropriate statistical test may have been a test for a statistical interaction. Specifically, the statistical test should assess if the association between fibrosis on biopsy and

Treatment If Extensive Fibrosis?
ESRD differs in young versus old patients or in diabetic versus other types of nephropathy.

While there is some uncertainty based on the statistical approach used, it is plausible that fibrosis would be less predictive of ESRD in both subgroups. There are significant structural changes in the kidney with normal aging including glomerulosclerosis, interstitial fibrosis, arteriosclerosis and cortical thinning [5]. Thus, some of the parenchymal damage seen on the biopsy of an older adult may be more reflective of aging. Considerations for the aging effects on the renal biopsy findings may help distinguish age-related and disease-related pathology. In particular, age-based reference limits for glomerulosclerosis based on living kidney donors have been developed and can be applied to the interpretation of renal biopsy findings in older adults [6]. Fibrosis may also be less predictive of ESRD in diabetic nephropathy due to its association with arteriosclerosis that may cause patchy fibrosis, making the amount of biopsy fibrosis less representative of the total kidney fibrosis.

This study has a number of important strengths. The study sample included good representation of African American and Hispanic patients who are disproportionally affected by kidney disease. The biopsies were read by 1 of 2 renal pathologists, limiting inter-rater source of variance. The authors did not use morphometric techniques to assess fibrosis, but this study is more applicable to clinical practice where pathologists rather than morphometry are used to assess the amount of fibrosis. Limitations are the single center without external validation. The relatively small sample size may have underpowered some of the subgroup analysis and did not allow for a more disease-specific analysis.

How should nephrologists interpret biopsy IFTA based on the results of this study? The study points out that although severe fibrosis is highly predictive of ESRD, patients can go years with severe fibrosis and not require dialysis. Even when prognosis is poor based on fibrosis severity, treatment should still be a consideration in select patients.

\section{Acknowledgments}

This paper was supported with funding from the National Institutes of Health, National Institute of Diabetes and Digestive and Kidney Diseases (DK090358).

\section{Disclosure Statement}

None. 


\section{References}

1 Nangaku M: Chronic hypoxia and tubulointerstitial injury: a final common pathway to end-stage renal failure. J Am Soc Nephrol 2006; 17:17-25.

$2 \mathrm{Lv}$ J, et al: Evaluation of the Oxford classification of IgA nephropathy: a systematic review and meta-analysis. Am J Kidney Dis 2013;62: 891-899.
3 Solez K, et al: Banff 07 classification of renal allograft pathology: updates and future directions. Am J Transplant 2008;8:753-760.

4 Menn-Josephy $\mathrm{H}$, et al: Renal interstitial fibrosis: an imperfect predictor of kidney disease progression in some patient cohorts. Am J Nephrol 2016;44:289-299.
5 Denic A, et al: Detection and clinical patterns of nephron hypertrophy and nephrosclerosis among apparently healthy adults. Am J Kidney Dis 2016;68:58-67.

6 Kremers WK, et al: Distinguishing age-related from disease-related glomerulosclerosis on kidney biopsy: the aging kidney anatomy study. Nephrol Dial Transplant 2015;30:20342039. 